\title{
Bioconversion of $p$-Tyrosol into Hydroxytyrosol under Bench-Scale Fermentation
}

\author{
Zouhaier Bouallagui (i) and Sami Sayadi \\ Laboratory of Environmental Bioprocesses, Centre of Biotechnology of Sfax, University of Sfax, P.O. Box “1177”, Sfax 3018, Tunisia \\ Correspondence should be addressed to Zouhaier Bouallagui; zouhair.bouallagui@cbs.rnrt.tn
}

Received 18 April 2018; Accepted 19 June 2018; Published 9 July 2018

Academic Editor: Yu-Guo Zheng

Copyright (C) 2018 Zouhaier Bouallagui and Sami Sayadi. This is an open access article distributed under the Creative Commons Attribution License, which permits unrestricted use, distribution, and reproduction in any medium, provided the original work is properly cited.

\begin{abstract}
Tyrosol hydroxylating Pseudomonas strain was previously isolated from olive mill wastewaters-irrigated soil. In the present work, experimental design was used to study the bioconversion of tyrosol in laboratory fermenters aiming at the recovery of the highest yields of hydroxytyrosol. The effects of biocatalyst loading and tyrosol concentration were studied. The bioconversion yield reached $86.9 \%$ (37.3 mM hydroxytyrosol) starting from a tyrosol concentration of $43 \mathrm{mM}$. Under these conditions, the specific productivity relative to the biocatalyst was $4.78 \mu \mathrm{M} / \mathrm{min} / \mathrm{g}$. The established model to predict bioconversion yield was validated in two bench-scale fermenters. At the downstream stage, the reaction product was recovered as a hydroxytyrosol rich solution after microfiltration and concentration under vacuum. Subsequent to this operation, hydroxytyrosol composition yielded $73.8 \%$ of the total dry matter.
\end{abstract}

\section{Introduction}

The bioactive properties of hydroxytyrosol have triggered the establishment of a new insight into its use as a naturally available antioxidant, mostly found in olive tree $[1,2]$. This later is thought to be a substitute to synthetic antioxidants in foods [3]. Indeed, this compound was demonstrated to prevent lipid peroxidation in many in vitro and in vivo models [4-6]. Synthetic pathways in the olive tree do not lead to the direct synthesis of hydroxytyrosol. Yet, this later mainly results from oleuropein hydrolysis taking place during olive fruit maturation [7]. Besides, this hydrolysis will be enhanced during the olive oil extraction process resulting in the increase in hydroxytyrosol concentration in olive mill wastewater, due to its low oil/water partition coefficient $[8$, 9]. This fact, associated with studies showing the beneficial activities of hydroxytyrosol, has promoted the development of efficient hydroxytyrosol recovery processes.

Despite the increasing demand for the use of hydroxytyrosol as a nutraceutical, there are still limited recovery processes that could be succeeded. Furthermore, although successful, these processes cannot satisfy the market requirements. Relevant recovery processes have used olive mill wastewater as a natural resource of hydroxytyrosol [10]. These processes were based on solvent extraction procedures. Two major drawbacks could be assigned for such procedures, which are the risk of solvent contamination and the need for additional chromatographic purification steps. The continuous countercurrent extraction system was suggested as the choice to partially overcome these problems [11]. However, the production yields were considered as unsatisfying.

During the last decade, few relevant processes based on bioconversion reactions were developed in order to produce hydroxytyrosol. Enzymatic processes using tyrosinase or $\beta$ glucosidase as biocatalysts and tyrosol or oleuropein as respective substrates have been reported [12-15]. In both cases, the purification of the enzyme rises as a major drawback. In addition, the heterogeneity of the reaction products or the use of a cofactor as ascorbic acid will further affect the product cost. Yet, a few numbers of researchers have reported the use of entire bacterial cells as effective catalysts for the recovery of biolabeled hydroxytyrosol. Bacterial strains belonging to Pseudomonas [16], Serratia [17], transformed E. coli [18], or Halomonas [19] strains were earlier identified as being capable of transforming tyrosol to hydroxytyrosol. The present work was designed to understand the laboratory fermentation process for the bioconversion of tyrosol into hydroxytyrosol through the elaboration of an experimental 
TABLE 1: Experimental design and results of the bioconversion of tyrosol into hydroxytyrosol. Numbers between parentheses represent the coded values of both studied variables.

\begin{tabular}{lccccc}
\hline Experiment & Biomass (g/L) & Tyrosol (g/L) & $\begin{array}{c}\text { Hydroxytyrosol production (mM) } \\
\text { Observed }\end{array}$ & $\begin{array}{c}\text { Residual hydroxytyrosol } \\
\text { Production }(\mathbf{m M})\end{array}$ \\
\hline 1 & $5.00(+1)$ & $6.00(+1)$ & 34.91 & 35.92 & -1.017 \\
2 & $5.00(+1)$ & $6.00(+1)$ & 37.39 & 35.92 & 1.462 \\
3 & $5.00(+1)$ & $5.00(0)$ & 29.88 & 29.93 & -0.056 \\
4 & $5.00(+1)$ & $5.00(0)$ & 29.48 & 29.93 & -0.454 \\
5 & $5.00(+1)$ & $4.00(-1)$ & 20.75 & 21.09 & -0.343 \\
6 & $5.00(+1)$ & $4.00(-1)$ & 21.50 & 21.09 & 0.408 \\
7 & $3.75(0)$ & $6.00(+1)$ & 33.61 & 33.22 & 0.387 \\
8 & $3.75(0)$ & $6.00(+1)$ & 32.39 & 33.22 & -0.832 \\
9 & $3.75(0)$ & $5.00(0)$ & 28.87 & 28.67 & 0.195 \\
10 & $3.75(0)$ & $5.00(0)$ & 28.61 & 28.67 & -0.064 \\
11 & $3.75(0)$ & $4.00(-1)$ & 20.66 & 21.27 & -0.614 \\
12 & $3.75(0)$ & $4.00(-1)$ & 22.20 & 21.27 & 0.928 \\
13 & $2.50(-1)$ & $5.00(0)$ & 30.9 & 29.96 & 0.934 \\
14 & $2.50(-1)$ & $5.00(0)$ & 29.41 & 29.96 & -0.555 \\
15 & $2.50(-1)$ & $4.00(-1)$ & 23.73 & 24.00 & -0.274 \\
16 & $2.50(-1)$ & $4.00(-1)$ & 23.9 & -24.00 & - \\
17 & $2.50(-1)$ & $6.00(+1)$ & - & - & - \\
18 & $2.50(-1)$ & $6.00(+1)$ & & - \\
\hline
\end{tabular}

design model involving the substrate and biomass concentrations as key variables that could influence the reaction using a bacterial strain isolated by our team.

\section{Materials and Methods}

2.1. Inoculum Preparation. Pseudomonas aeruginosa strain, previously isolated and identified in our laboratory [16], was cultivated in Lauria broth in one litre Erlenmeyer flasks inoculated from solid media (1.5\% agar). For the induction of tyrosol metabolizing pathway, filter sterilized tyrosol solution was added to the culture at a final concentration of $0.6 \mathrm{~g} / \mathrm{L}$. The culture was then allowed to grow overnight at $30^{\circ} \mathrm{C}$ with orbital shaking at $180 \mathrm{rpm}$.

2.2. Biomass Production. The biomass used for the conversion of tyrosol into hydroxytyrosol was grown in mineral medium (containing in $\mathrm{g} / \mathrm{L}$ ): $\mathrm{Na}_{2} \mathrm{HPO}_{4}, 2.44 ; \mathrm{KH}_{2} \mathrm{PO}_{4}, 1.52$; $\left(\mathrm{NH}_{4}\right)_{2} \mathrm{SO}_{4}, 1.5 ; \mathrm{MgSO}_{4}, 7 \mathrm{H}_{2} \mathrm{O}, 0.2 ; \mathrm{CaCl}_{2}, \mathrm{H}_{2} \mathrm{O}, 0.05$ and 10 $\mathrm{ml}$ of a trace-element solution containing ( in g/L): EDTA, 0.5; FeSO4, 0.2; $\mathrm{ZnSO}_{4}, 7 \mathrm{H}_{2} \mathrm{O}, 0.01 ; \mathrm{MnCl}_{2}, 4 \mathrm{H}_{2} \mathrm{O}, 0.003$; $\mathrm{Na}_{2} \mathrm{MoO}_{4}, 2 \mathrm{H}_{2} \mathrm{O}, 0.003 ; \mathrm{H}_{3} \mathrm{BO}_{3}, 0.03 ; \mathrm{CuCl}_{2}, 6 \mathrm{H}_{2} \mathrm{O}, 0.002$; $\mathrm{NiCl}_{2}, 6 \mathrm{H}_{2} \mathrm{O}, 0.002$; and $\mathrm{CoCl}_{2}, 6 \mathrm{H}_{2} \mathrm{O}, 0.02$. The $\mathrm{pH}$ of the medium was adjusted to 7.2. Tyrosol was used at $1 \mathrm{~g} / \mathrm{L}$ as a unique carbon source. Two fermentation types (3.6 L and 7.5 L fermenters, INFORS AG CH-4103 Bottmingen/Switzerland) were inoculated with the induced inoculum. The biomass was grown at $30^{\circ} \mathrm{C}$ and the agitation and the aeration in the fermenters were adjusted in order to keep the oxygen saturation rate in the range of $30 \%$. Bacterial growth was followed spectrophotometrically until the late logarithmic phase. At this stage, the culture was collected by centrifugation $(5500 \times \mathrm{g}$ at $4^{\circ} \mathrm{C}$ for $10 \mathrm{~min}$ ) and washed twice with $\mathrm{M} 9$ phosphate buffered saline (4.2 mM Na $\mathrm{mPO}_{4}, 2.2 \mathrm{mM} \mathrm{KH} \mathrm{PO}_{4}, 0.9$ $\mathrm{mM} \mathrm{NaCl}$, and $1.9 \mathrm{mM} \mathrm{NH}_{4} \mathrm{Cl}$ ). The biomass was again resuspended in $\mathrm{M} 9$ phosphate buffered saline as bioconversion medium. Final biomass and tyrosol concentrations were chosen following the experimental design (Table 1). All runs were performed in $2 \mathrm{~L}$ working volume (3.6 L fermenter).

2.3. Experimental Design and Optimization. Response surface methodology using a central composite design was adopted to determine the effect of biomass and substrate on the production of hydroxytyrosol. Three coded values were fixed for each of the continuous variables. These coded values were calculated according to the equation

$$
x_{i}=\frac{X_{i}-X_{0}}{\Delta X}
$$

$x_{i}$ is the coded value of the independent variable, $X_{i}$ the natural value of the independent variable, $X_{0}$ the natural value of the independent variable at the centre point, and $\Delta X$ the step change value ( $\Delta X$ is 1.25 for the biomass concentration and 1 for the substrate concentration). A set of 18 experiments was generated by the experimental design (Table 1).

The response (hydroxytyrosol production (mM)) was correlated with the independent variables (biomass and tyrosol concentrations) through a second-order polynomial equation:

$$
Y=b_{0}+b_{1} x_{1}+b_{2} x_{2}+b_{11} x_{1}^{2}+b_{22} x_{2}^{2}+b_{12} x_{1} x_{2}
$$


TABLE 2: Analysis of variance (ANOVA) for the predicted quadratic model.

\begin{tabular}{|c|c|c|c|c|c|}
\hline Source & Sum of squares & DF & Mean square & F-value & Probability $p$ \\
\hline Model & 414.02 & 5 & 82.804 & 117.23 & $<.0001$ \\
\hline Lack of fit & 0.53 & 2 & 0.266 & 0.326 & 0.73 \\
\hline Pure error & 6.530 & 8 & 0.816 & & \\
\hline Corrected total & 421.08 & 15 & & & \\
\hline
\end{tabular}

where $Y$ is the hydroxytyrosol production (mM); $x_{1}$ and $x_{2}$ are the coded values of the biomass and tyrosol concentration, respectively. $b_{0}$ is the intercept; $b_{1}$ and $b_{2}$ are linear coefficients for biomass and tyrosol, respectively; $b_{11}$ and $b_{22}$ are squared coefficients and $b_{12}$ is the interaction coefficient. Data were analyzed using JMP 9.0.2 software.

2.4. Analytical Method. Throughout the bioconversion time, samples $(1 \mathrm{~mL})$ were withdrawn periodically (every 2 hours or less depending on the progress of the reaction) and centrifuged at $8000 \times \mathrm{g}$ for $10 \mathrm{~min}$. The supernatant was analyzed using high performance liquid chromatography (HPLC) to determine the concentration of the different metabolites. The instrument consisted of a Shimadzu C-R6A liquid chromatograph coupled to a Shimadzu SPD-6A UV detector. The separation was carried out on a C18 reverse phase column $(250 \mathrm{~mm} \times 4.6 \mathrm{~mm}$; $5 \mu \mathrm{m}$ particle size; Shimpack VP-ODS). The mobile phase consisted of (A) $0.1 \%$ formic acid in water and (B) $70 \%$ acetonitrile in water. The following gradient was used: 0 min 10\% B, $25 \min 25 \% \mathrm{~B}, 35$ $\min 80 \%$ B, $37 \min 100 \%$ B, $40 \mathrm{~min} 100 \%$ B, and $50 \min 10 \%$ $B$. The flow rate was set at $0.7 \mathrm{~mL} / \mathrm{min}$. The injection volume was $20 \mu \mathrm{L}$. The eluted compounds were detected at $\lambda 280 \mathrm{~nm}$ and identified by comparison with authentic standards.

\section{Results and Discussion}

3.1. Predictive Model of Hydroxytyrosol Production. We previously studied the bioconversion of tyrosol into hydroxytyrosol in Erlenmeyer flasks [16]. In the present work, we are focusing on the scale-up of the reaction into laboratory fermenters. In order to examine the hydroxytyrosol production $(\mathrm{mM})$, two independent variables, biomass and tyrosol concentrations, were investigated using a response surface methodology. The biomass and tyrosol concentrations were varied in the ranges of 2.5 to $5 \mathrm{~g}$ (wet weight)/L and 4 to 6 $\mathrm{g} / \mathrm{L}$ (28.9 to $43.4 \mathrm{mM})$, respectively. These two variables were investigated through the use of a central composite design. The experimental design generated 18 experiments among which only 16 runs were considered and the two others were discarded due to an excessive lengthening of the reaction time (Table 1). For all of the sixteen experiments, reaction time ranged between 18 and 27 hours. However, for experiments 17 and 18, reaction times lasted for more than 50 hours. Table 1 shows that the highest hydroxytyrosol production was obtained at $5 \mathrm{~g} / \mathrm{L}$ biomass and $6 \mathrm{~g} / \mathrm{L}$ tyrosol (experiment $\mathrm{N}^{\circ} 2$ ). Under these working concentrations, the molar bioconversion yield was brought to $86.11 \%$ with $37.39 \mathrm{mM}$ hydroxytyrosol production. A representative time course evolution of the bioconversion reaction is given in Figure 1.

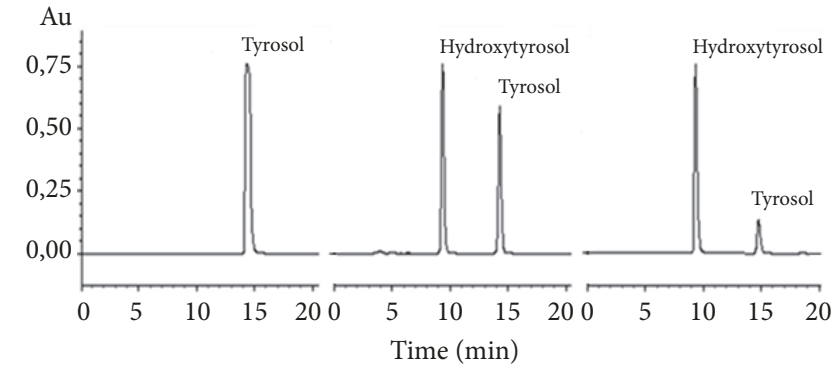

(a)

(b)

(c)

FIGURE 1: Illustrative time course chromatograms for the bioconversion of tyrosol into hydroxytyrosol. (a) HPLC spectrum of bioconversion medium at starting time; (b) composition of reaction medium at reaction mid-time; (c) composition of bioconversion medium at a late stage of the reaction.

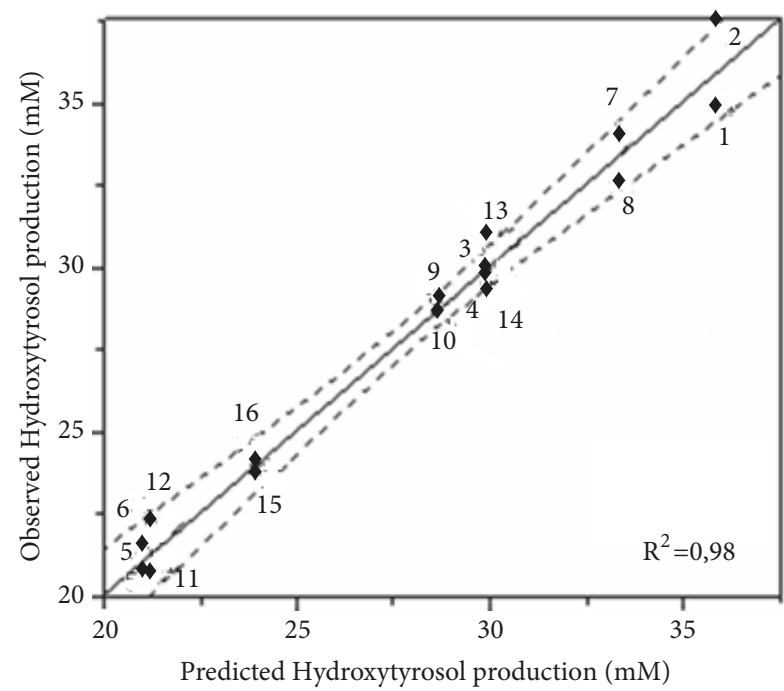

FIGURE 2: Correlation between experimental and predicted response values for hydroxytyrosol production. Numbers correspond to the experiment number given in Table 1. Dashed line: 0.05 significance curve. Continuous line: line of fit.

The analysis of variance (ANOVA) and the lack of fit of the predictive model are shown (Table 2). Analysis was based on $p$ values as a criterion in order to check the significance of the model $[20,21]$. The model F-value of 117.235 associated with a $p$ value $<0.0001$ implies that the model is highly significant. In fact, this probability denotes that the noise error is less than $0.01 \%$. Another marker of the goodness of our model is the regression coefficient $R^{2}$. A coefficient $R^{2}=0.983$ indicates that only $1.7 \%$ of the experimental data cannot be explained by the predicted model (Figure 2). The 
TABLE 3: Estimated values of the model coefficients.

\begin{tabular}{lccr}
\hline Model coefficients & Estimate & Standard Error & $P$-value \\
\hline$b_{0}$ & 28.674 & 0.467 & $<.0001$ \\
$b_{1}$ & -0.012 & 0.330 & 0.9697 \\
$b_{2}$ & 5.974 & 0.330 & $<.0001$ \\
$b_{12}$ & 1.439 & 0.449 & 0.0094 \\
$b_{11}$ & 1.277 & 0.476 & 0.0230 \\
$b_{22}$ & -1.426 & 0.476 & 0.0135 \\
\hline
\end{tabular}

lack of fit (0.326) with a probability of 0.730 implies that it is insignificant relatively to the pure error.

The calculated coefficient values of the predicted model and their significance are shown in Table 3 . Through the analysis of the $p$ values it is shown that the biomass linear coefficient $\left(b_{1}\right)$ is not significant either at $1 \%$ or at $5 \%$. Nevertheless, for the same variable, the quadratic $\left(b_{11}\right)$ and the cross-product $\left(b_{12}\right)$ coefficients are still significant at $5 \%$. Furthermore, the substrate linear coefficient $\left(b_{2}\right)$ is highly significant at $1 \%$. Finally, the quadratic coefficient $b_{22}$ seems to be insignificant at $1 \%$ confidence interval. These data suggest that the biomass loading does not have a significant effect on hydroxytyrosol production. Nonetheless, biomass concentration does have an effect only on the reaction time (data not shown). This fact was understood after calculating the second order polynomial model, which could clearly explain the effect of the studied variables on hydroxytyrosol production expressed in the following equation:

$$
\begin{aligned}
Y= & -3.729-11.895 X_{1}+15.917 X_{2}+0.817 X_{1}^{2} \\
& -1.426 X_{2}^{2}+1.151 X_{1} X_{2}
\end{aligned}
$$

where $Y$ represents the hydroxytyrosol production and $\mathrm{X}_{1}$ and $\mathrm{X}_{2}$ are biomass and tyrosol concentrations $(\mathrm{g} / \mathrm{L})$, respectively.

3.2. Experimental Model Validation. For the model validation, a couple of biomass and tyrosol concentrations $(\mathrm{g} / \mathrm{L})$ was chosen $(4.25 ; 5.5)$. For these concentrations, the model predicted a hydroxytyrosol production of $31.81 \mathrm{mM}$. In order to validate the model, two duplicated experiments were run in two different fermenters (3.6 L and 7.5 L). Under these conditions, the experimental hydroxytyrosol production values were 32.16 and $31.08 \mathrm{mM}$ in both fermenters, respectively. The corresponding reaction times for both fermenters were 24.33 and 25.25 hours. Therefore, these data clearly show that the reaction times for the validation experiments are still within the range of all other experiments (18 to 27 hours) and the experimental hydroxytyrosol production values are in agreement with those predicted by the model.

Despite the biological importance of hydroxytyrosol as a potent antioxidant molecule, which might be implicated in the prevention of oxidative stress related diseases, its recovery is still challenging. Processes including chemical synthesis and recovery from olive by-products (leaves or milling wastewater) are not without limitations [10, 22]. Recently, several biological processes have been reported.
These bioprocesses include either enzymatic or microbial catalysts $[16,17,19,23]$. Within the present work, we showed that the bacterial recovery of hydroxytyrosol could be interesting considering the highest achievable yield and the scale-up feasibility of the bioconversion process.

3.3. Hydroxytyrosol Recovery. The recovery and probably the purification of the bioconversion product are considered as ultimate stages in the reaction process. The majority of the available hydroxytyrosol recovery processes are based on chromatography purification either from olive by-products or biological (enzymatic or cellular) and chemical reactions. Within the present process, the reaction product was recovered after the first step of centrifugation at $8000 \times \mathrm{g}$ for 10 minutes, followed by a microfiltration $(0.22 \mu \mathrm{m})$ in order to eliminate any traces of microorganisms. The filtrate was then concentrated under vacuum using a rotatory evaporator. A 60 -fold concentrated product was obtained. This step will allow the precipitation of a major part of the salts present in the reaction medium. Subsequently, characterization by HPLC for qualitative and quantitative analysis of hydroxytyrosol and other potential metabolites was performed. Results showed that the concentrated solution contained $16.7 \%(\mathrm{w} / \mathrm{v})$ hydroxytyrosol, which was equivalent to $73.8 \%$ of the total dry matter. To our knowledge, this composition could offer the highest percentage of biolabeled hydroxytyrosol yet available on the market. Thereafter, a single chromatographic step or the application of membranes would allow the recovery of high purity hydroxytyrosol. In particular, the use of membrane technology would result in an efficient selectivity towards target molecules. For this reason, membranes were the option when purifying hydroxytyrosol from olive mill wastewaters [24-26].

\section{Conclusion}

Considering the above shown data, it can be advanced that the fermentation process adopted for the bioconversion of $p$ tyrosol into hydroxytyrosol might be encouraging for transferring this reaction to the pilot scale and potentially using a safer engineered microorganism. In addition, response surface methodology will always be a prosperous approach to investigate variables interactions when many are considered.

\section{Data Availability}

The data used to support the findings of this study are available from the corresponding author upon request. 


\section{Conflicts of Interest}

The authors declare that they have no conflicts of interest.

\section{Acknowledgments}

This work was financially supported by the Tunisian Ministry of Higher Education and Scientific Research under contract program of the Laboratory of Environmental Bioprocesses at Centre of Biotechnology of Sfax.

\section{References}

[1] J. G. Fernández-Bolaños, Ó. López, J. Fernández-Bolaños, and G. Rodríguez-Gutiérrez, "Hydroxytyrosol and derivatives: Isolation, synthesis, and biological properties," Current Organic Chemistry, vol. 12, no. 6, pp. 442-463, 2008.

[2] Z. Bouallagui, M. Bouaziz, S. Lassoued, J. M. Engasser, M. Ghoul, and S. Sayadi, "Hydroxytyrosol acyl esters: Biosynthesis and activities," Applied Biochemistry and Biotechnology, vol. 163, no. 5, pp. 592-599, 2011.

[3] S. Tejada, S. Pinya, M. d. Mar Bibiloni, J. A. Tur, A. Pons, and A. Sureda, "Cardioprotective Effects of the Polyphenol Hydroxytyrosol from Olive Oil," Current Drug Targets, vol. 18, no. 13, 2017.

[4] R. Aparicio, L. Roda, M. A. Albi, and F. Gutiérrez, "Effect of various compounds on virgin olive oil stability measured by Rancimat," Journal of Agricultural and Food Chemistry, vol. 47, no. 10, pp. 4150-4155, 1999.

[5] T. Hu, X.-W. He, J.-G. Jiang, and X.-L. Xu, "Hydroxytyrosol and its potential therapeutic effects," Journal of Agricultural and Food Chemistry, vol. 62, no. 7, pp. 1449-1455, 2014.

[6] S. Pan, L. Liu, H. Pan et al., "Protective effects of hydroxytyrosol on liver ischemia/reperfusion injury in mice," Molecular Nutrition \& Food Research, vol. 57, no. 7, pp. 1218-1227, 2013.

[7] I. Hassen, H. Casabianca, and K. Hosni, "Biological activities of the natural antioxidant oleuropein: exceeding the expectation-a mini-review," Journal of Functional Foods, 2014.

[8] R. Sacchi, A. Paduano, F. Fiore, D. D. Medaglia, M. L. Ambrosino, and I. Medina, "Partition behavior of virgin olive oil phenolic compounds in oil-brine mixtures during thermal processing for fish canning," Journal of Agricultural and Food Chemistry, vol. 50, no. 10, pp. 2830-2835, 2002.

[9] P. S. Rodis, V. T. Karathanos, and A. Mantzavinou, "Partitioning of olive oil antioxidants between oil and water phases," Journal of Agricultural and Food Chemistry, vol. 50, no. 3, pp. 596-601, 2002.

[10] A. Bonetti, S. Venturini, A. Ena, and C. Faraloni, "Innovative method for recovery and valorization of hydroxytyrosol from olive mill wastewaters," Water Science and Technology, vol. 74, no. 1, pp. 73-86, 2016.

[11] N. Allouche, I. Fki, and S. Sayadi, "Toward a high yield recovery of antioxidants and purified hydroxytyrosol from olive mill wastewaters," Journal of Agricultural and Food Chemistry, vol. 52, no. 2, pp. 267-273, 2004.

[12] R. Briante, F. La Cara, M. P. Tonziello, F. Febbraio, and R. Nucci, "Antioxidant activity of the main bioactive derivatives from oleuropein hydrolysis by hyperthermophilic $\beta$-glycosidase," Journal of Agricultural and Food Chemistry, vol. 49, no. 7, pp. 3198-3203, 2001.
[13] R. Briante, M. Patumi, F. Febbraio, and R. Nucci, "Production of highly purified hydroxytyrosol from Olea europaea leaf extract biotransformed by hyperthermophilic $\beta$-glycosidase," Journal of Biotechnology, vol. 111, no. 1, pp. 67-77, 2004.

[14] M. Brouk, Y. Nov, and A. Fishman, "Improving biocatalyst performance by integrating statistical methods into protein engineering," Applied and Environmental Microbiology, vol. 76, no. 19, pp. 6397-6403, 2010.

[15] J. C. Espín, C. Soler-Rivas, E. Cantos, F. A. Tomás-Barberán, and H. J. Wichers, "Synthesis of the antioxidant hydroxytyrosol using tyrosinase as biocatalyst," Journal of Agricultural and Food Chemistry, vol. 49, no. 3, pp. 1187-1193, 2001.

[16] N. Allouche, M. Damak, R. Ellouz, and S. Sayadi, "Use of Whole Cells of Pseudomonas aeruginosa for Synthesis of the Antioxidant Hydroxytyrosol via Conversion of Tyrosol," Applied and Environmental Microbiology, vol. 70, no. 4, pp. 2105-2109, 2004.

[17] N. Allouche and S. Sayadi, "Synthesis of hydroxytyrosol, 2-hydroxyphenylacetic acid, and 3-hydroxyphenylacetic acid by differential conversion of tyrosol isomers using Serratia marcescens strain," Journal of Agricultural and Food Chemistry, vol. 53, no. 16, pp. 6525-6530, 2005.

[18] E. Orenes-Piñero, F. García-Carmona, and Á. Sánchez-Ferrer, "A new process for obtaining hydroxytyrosol using transformed Escherichia coli whole cells with phenol hydroxylase gene from Geobacillus thermoglucosidasius," Food Chemistry, vol. 139, no. 1-4, pp. 377-383, 2013.

[19] P.-P. Liebgott, M. Labat, L. Casalot, A. Amouric, and J. Lorquin, "Bioconversion of tyrosol into hydroxytyrosol and 3,4-dihydroxyphenylacetic acid under hypersaline conditions by the new Halomonas sp. strain HTB24," FEMS Microbiology Letters, vol. 276, no. 1, pp. 26-33, 2007.

[20] Y.-L. Gao and X.-R. Ju, "Statistical prediction of effects of food composition on reduction of Bacillus subtilis As 1.1731 spores suspended in food matrices treated with high pressure," Journal of Food Engineering, vol. 82, no. 1, pp. 68-76, 2007.

[21] S. Greenland, S. J. Senn, K. J. Rothman et al., "Statistical tests, $\mathrm{P}$ values, confidence intervals, and power: a guide to misinterpretations," European Journal of Epidemiology, vol. 31, no. 4, pp. 337-350, 2016.

[22] R. Capasso, A. Evidente, S. Avolio, and F. Solla, "A highly convenient synthesis of hydroxytyrosol and its recovery from agricultural waste waters," Journal of Agricultural and Food Chemistry, vol. 47, no. 4, pp. 1745-1748, 1999.

[23] Z. Bouallagui and S. Sayadi, "Production of high hydroxytyrosol yields via tyrosol conversion by Pseudomonas aeruginosa immobilized resting cells," Journal of Agricultural and Food Chemistry, vol. 54, no. 26, pp. 9906-9911, 2006.

[24] C. A. Paraskeva, V. G. Papadakis, D. G. Kanellopoulou, P. G. Koutsoukos, and K. C. Angelopoulos, "Membrane filtration of olive mill wastewater and exploitation of its fractions," Water Environment Research, vol. 79, no. 4, pp. 421-429, 2007.

[25] F. Bazzarelli, E. Piacentini, T. Poerio, R. Mazzei, A. Cassano, and L. Giorno, "Advances in membrane operations for water purification and biophenols recovery/valorization from OMWWs," Journal of Membrane Science, vol. 497, pp. 402-409, 2016.

[26] M. Cockrem and C. C. Santana, "Downstream processing of bioproducts," Applied Biochemistry and Biotechnology - Part A Enzyme Engineering and Biotechnology, vol. 108, no. 1-3, pp. 827828, 2003. 


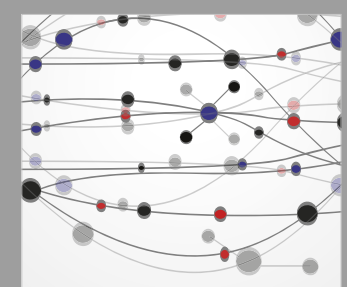

The Scientific World Journal
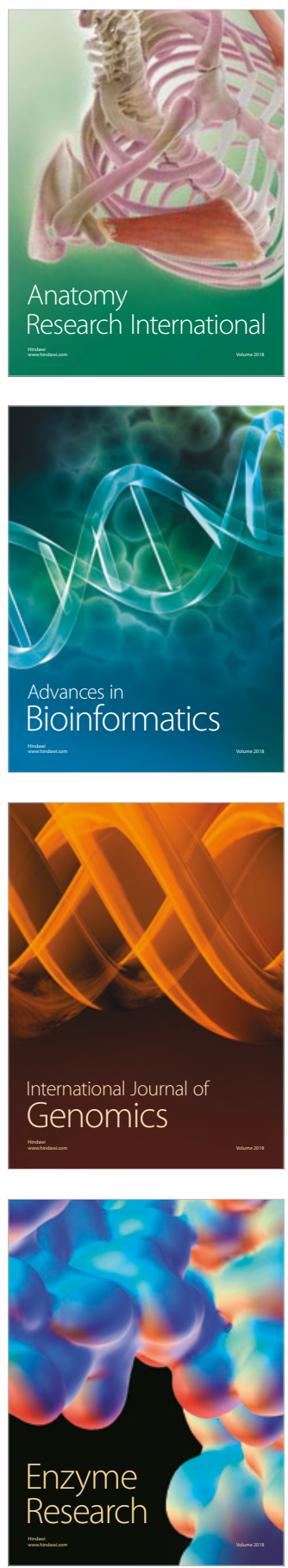
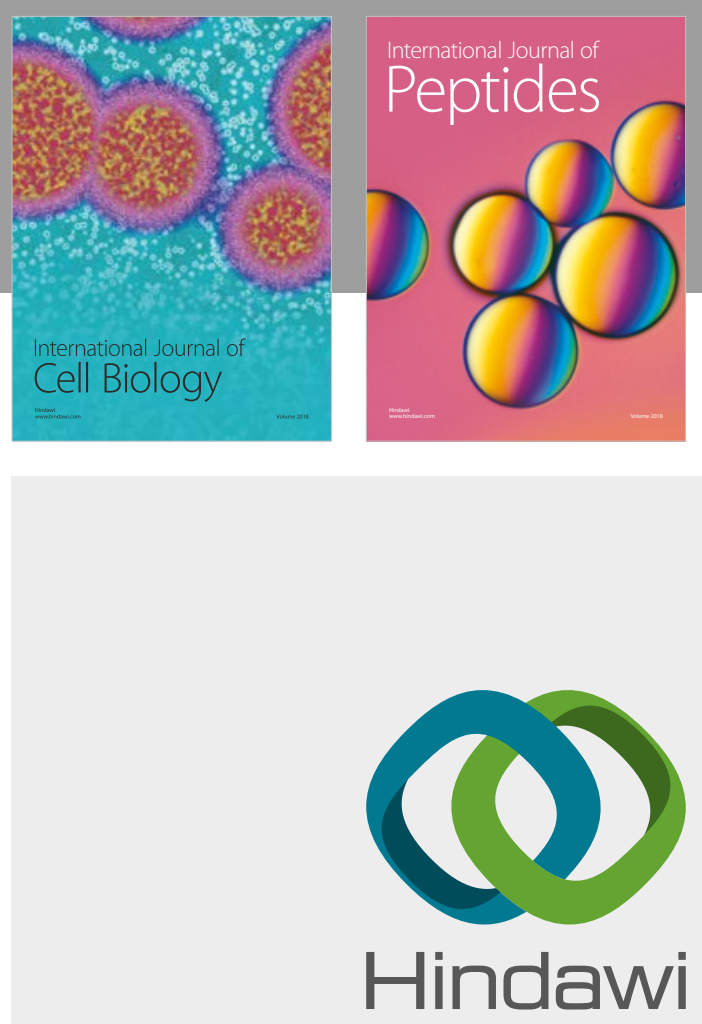

Submit your manuscripts at

www.hindawi.com
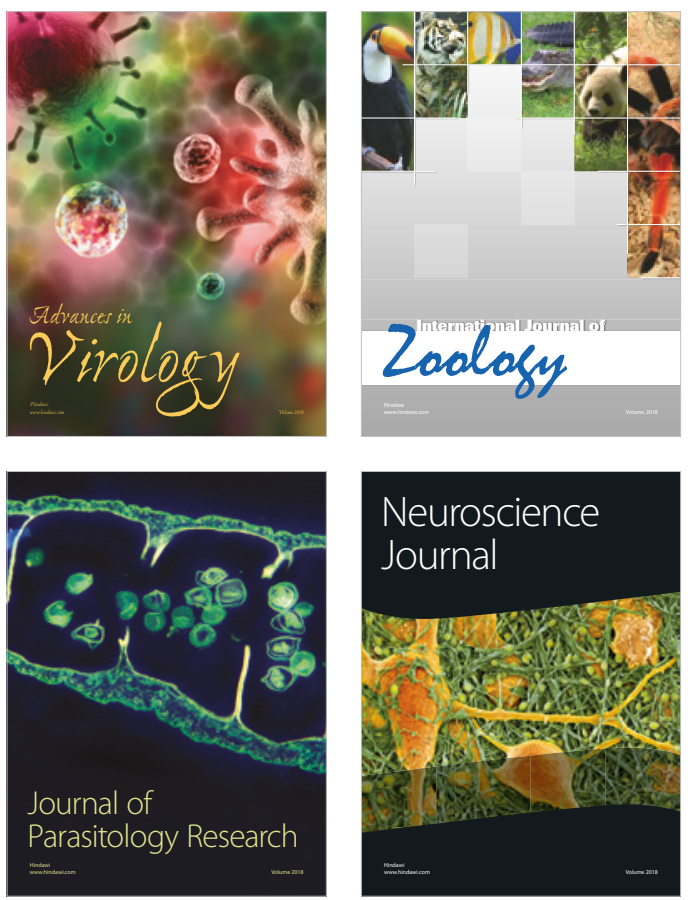
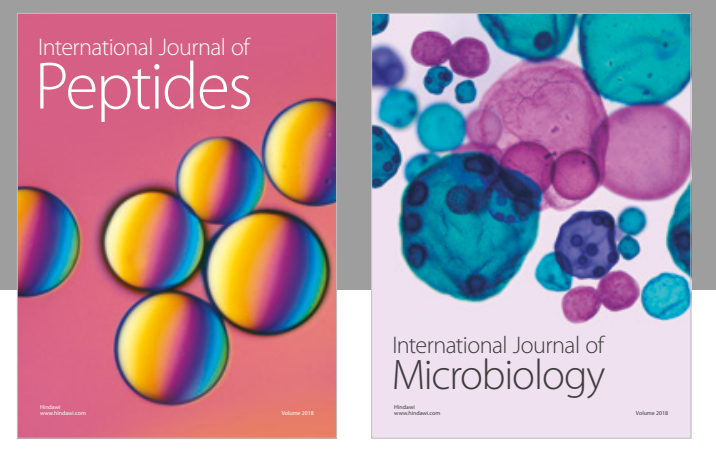

nternational Journal of Microbiology
Journal of
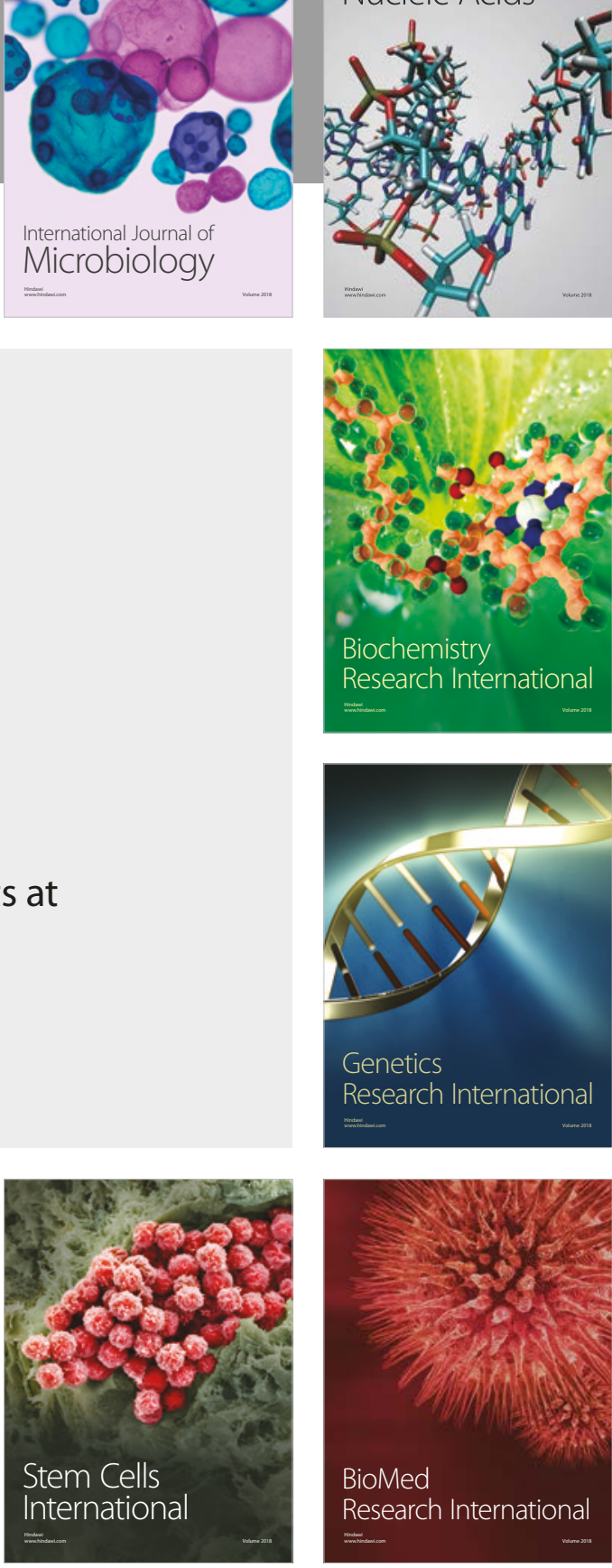
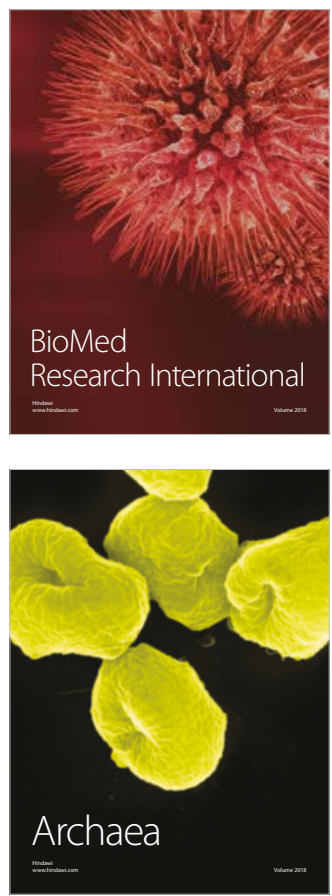